\title{
Different Applications of Magnetic Nanoparticles in the Rapid Monitoring of Ochratoxin A
}

\author{
AZAM SARGAZI and MOSTAFA HEIDARI MAJD* \\ Department of Medicinal Chemistry, Faculty of Pharmacy, \\ Zabol University of Medical Sciences, Zabol, Iran \\ ${ }^{*}$ Corresponding author E-mail: mostafamajd@ @ live.com
}

http://dx.doi.org/10.13005/ojc/330141

(Received: January 03, 2017; Accepted: February 04, 2017)

\begin{abstract}
Toxic effects of mycotoxins are still an unresolved subject for health of humans and animals. Therefore, several methods has been created to determine and eliminate mycotoxins from foodstuffs, and also are developing. For instance, utilization of magnetic nanoparticles (MNPs) when are combined with different analytical tools is one of the developing methods which could be useful for extraction of mycotoxins. As in some methods, they were employed as newfound adsorbents in magnetic solid phase extraction approaches. In some other methods, MNPs were used for immobilization of antibodies to enhance the efficiency of the analytical tools such as square wave voltammetry. Or in another method, amine-functionalized MNPs were reacted with ochratoxin A (OTA) to enhance performance of chemiluminescence immunoassay. Results of all methods were showed that utilizing of MNPs could reduce cost, easy preparation, and increase the stability, speed, sensitivity, precision and reproducibility. So, these new methods have elicited great hopes to be suitable alternative for older methods.
\end{abstract}

Keywords: Ochratoxin A, Extraction, Magnetic Solid Phase Extraction, Aptamer-Targeted Magnetic Nanospheres, Chemiluminescence Immunoassay.

\section{INTRODUCTION}

Molds are filamentous fungi which found in many feedstuffs, and produce toxins called mycotoxins. However, mycotoxins are classified as secondary metabolites and their function are not related to the mold's existence, but they can affect the health of animals when they consume from these contaminated feeds ${ }^{1}$. Mold growth can occur during storage and also during feed processing under warm and humid conditions. Therefore, mycotoxin production could be occur in un-cleaned silos, shipment systems and also food factory; so it is possible that more than one mycotoxin exist in every infected feeds ${ }^{2,3}$. 
On the other hand, many mycotoxins are known which their toxin production and also their structural chemistry depend on the susceptibility, metabolism and defense mechanisms ${ }^{4}$. So, they remain in the food chain, even in cooking and freezing treatments. Mycotoxins also resist dissociation in digestive tract which can have a noticeable effect on immune system of animals and humans ${ }^{5,6}$.

Several mycotoxins which can contaminate foods are included ochratoxins, aflatoxins, zearalenone and deoxynivalenol ${ }^{7}$. Meanwhile, Ochratoxins are derivative of isocoumarin which produced by several fungi belonging to Aspergillus or Penicillium species ${ }^{8}$. Ochratoxins, based on chemical structures, can be divided into three secondary metabolite forms, including $A, B$, and $\mathrm{C}^{2}$; but Ochratoxin A (OTA) is a prevalent and toxic component of this family which can affect mainly the kidney ${ }^{9}$. OTA have been discovered mainly in grape juice, coffee, cereal, milk, beer, pulses, spices, dried fruits and also meat products ${ }^{10,11}$. On the other hand, OTA can be the reason of serious diseases in humans such as hepatotoxic, nephrotoxic, immunotoxic, etc, because of its strong affinity to serum albumin, stability against acidic degradation and also stability during food processing operations ${ }^{12}$. Therefore, it is important that its amount be measured by an analysis method with well detection limit.

\section{Comparison of Analytical Methods used for Detection of Ochratoxin A}

Various methods have been reported for laboratory assessment of ochratoxin $A$ in food samples, including high-performance liquid chromatography (HPLC), capillary electrophoresis (CE), gas chromatography-mass spectrometry (GCMS), thin layer chromatography (TLC), enzyme-linked immunosorbent assay (ELISA), fluorimetry (FL), immunoassay based on fluorescence polarization (FPIA), and immunosensor based on surface plasmon resonance $(\mathrm{SPR})^{13}$. One of the most common approaches among these mentioned methods for detection of OTA is the HPLC with fluorescence detector (HPLC/FLD) in which OTA must be extracted by the immunoaffinity column (IAC) ${ }^{14}$. Briefly in this method, the sample solutions are passed through the columns filled with immobilized antibody against the ochratoxin A. Sample matrix is washed out by water or an aqueous buffer and OTA is gathered by methanol ${ }^{15}$. Though this method is sensitive and selective, but has some disadvantages such as time-consuming, high cost, and contamination of IAC with the ethyl ester residue of OTA which extensively reduce its efficiency ${ }^{14}$.

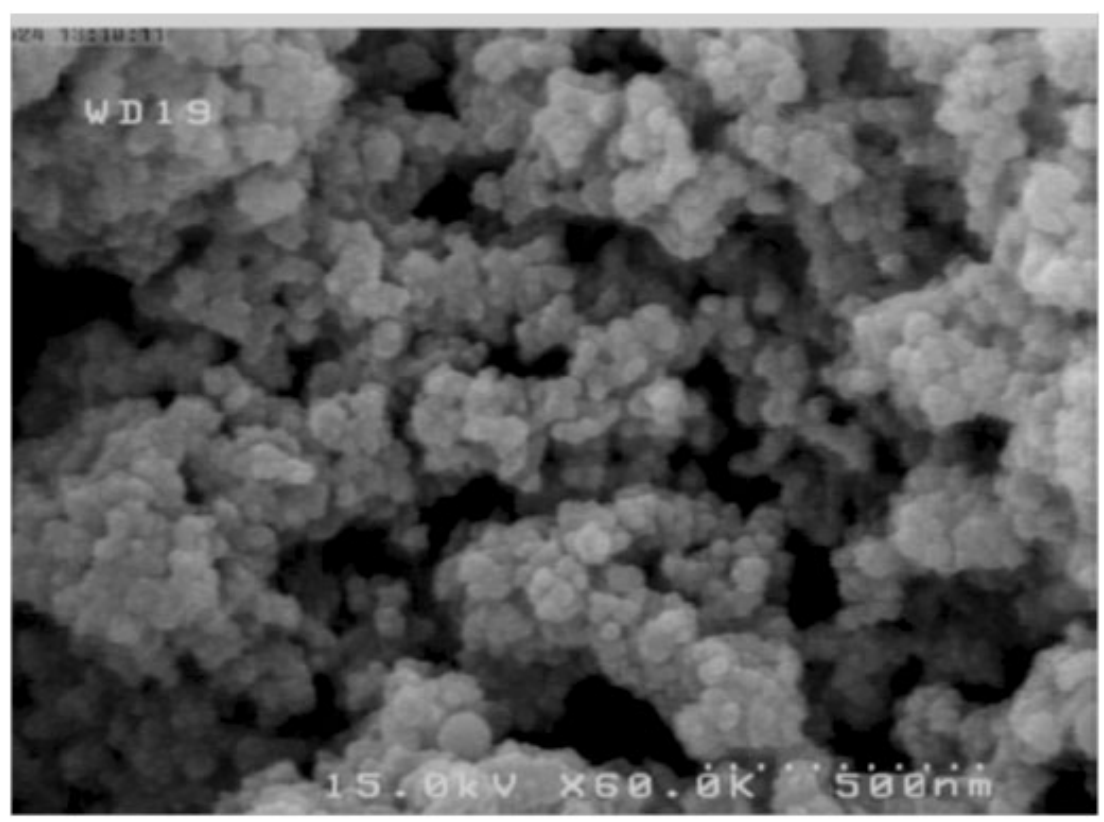

Fig. 1: SEM micrograph of EGBMA-MSPT-MNPs (WD19) sorbents 
Over the last decade, some alternative methods have been created for extraction and detection of ochratoxin $A$, but none have been so successful. For instance, TLC and CE methods aren't very sensitive, the FL method has higher sensitivity than that of the TCL, but its selectivity is low, ${ }^{16}$ and in ELISA method, due to the poor chemical/physical stability of the antibodies or enzymes, their use in acidic or basic conditions and also organic solvents are limited ${ }^{17}$. Therefore, the investigation of the modern methods, such as magnetic solid phase extraction (MSPE) which has got many benefits such as low toxicity, simple preparation and low price, is necessary for detection of OTA ${ }^{14}$.

Magnetic nanoparticles have some advantages such as suitable nano-scale size, high hydrophilicity and more surface modification with functionalized groups, which make them a good candidate for use in extraction ${ }^{18,19}$. Also, other abilities like large surface area to the volume ratio, rapid extraction and high extraction efficiencies, could be helpful for this purpose. So, in this paper, we review all the methods in which MNPs are used for extraction and measurement of OTA in foods and biological fluids.

\section{Detection of Ochratoxin A by Magnetic Solid Phase Extraction}

A novel, fast, simple, and environmentally friendly method based on modified $\mathrm{Fe}_{3} \mathrm{O}_{4}$ nanoparticles was introduced for extraction of OTA by Mashhadizadeh et al., at $2013^{20}$. In this method, a HPLC-fluorescence detection (Waters 474) system with RP-18e column was employed for determination of OTA after extraction by MNPs. For this purpose, $\mathrm{Fe}_{3} \mathrm{O}_{4}$ nanoparticles were synthesized and then were modified with 3-(trimethoxysilyl)-1-propanethiol (MSPT) and ethylene glycol bis-mercaptoacetate (EGBMA), respectively. Figure 1 represents the SEM micrograph of EGBMA-MSPT-MNPs sorbents with average size around $\sim 40 \mathrm{~nm}$.

Usual step for the extraction of OTA, before employing the HPLC, is immunoaffinity column (IAC) clean-up procedure which has some disadvantages ${ }^{21}$. But, in this work, MNPs were selected instead IACs to provide a successful interaction with OTA. The OTA
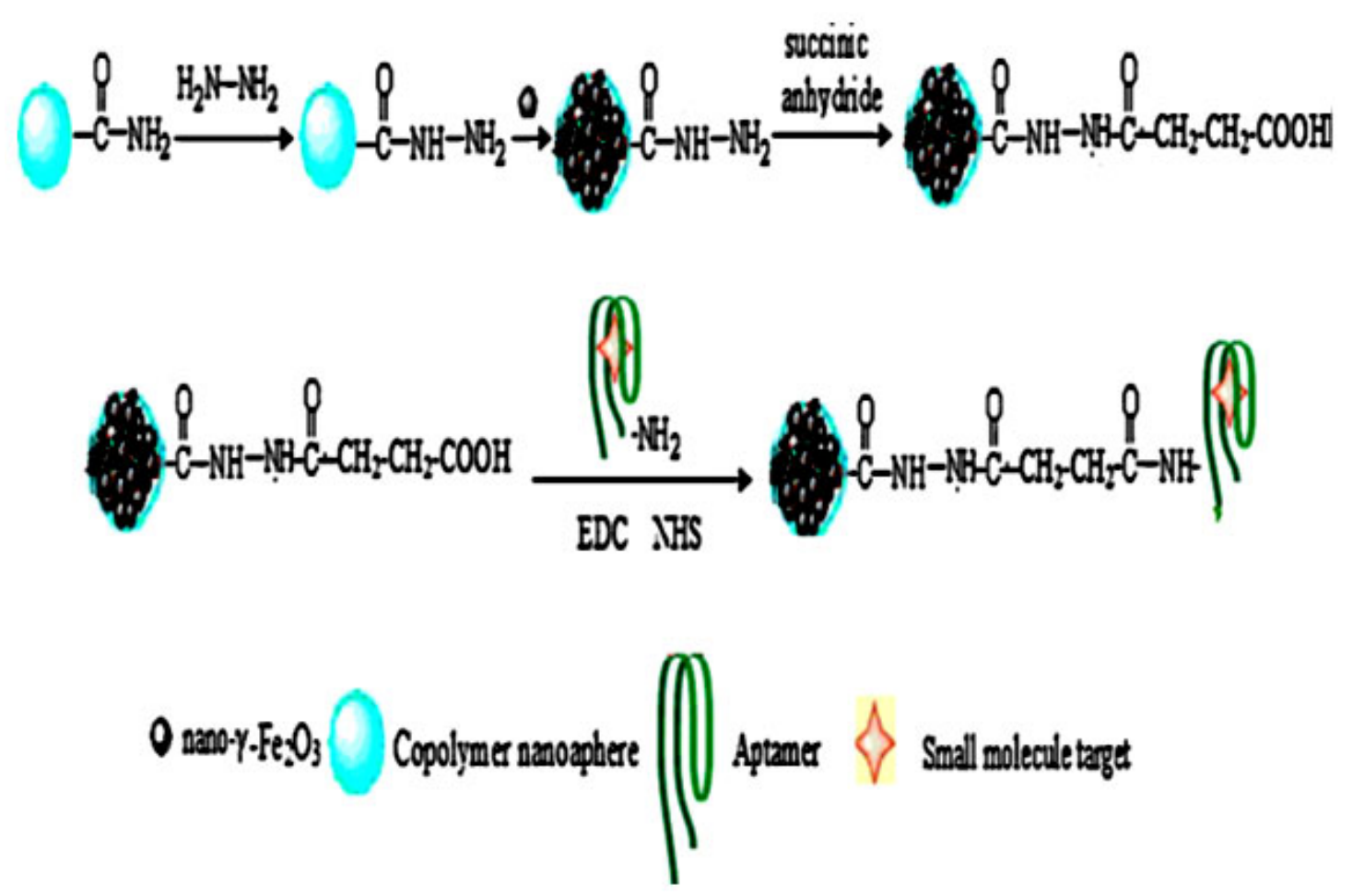

Fig. 2: Schematically demonstrates fabrication of the MNS-aptamer adsorbents 
as a polar molecule contains both ionizable and hydrophobic sections, therefore authors hypothesize that magnetic adsorbents (EGBMA-MSPT-MNPs) with both electrostatic and hydrophobic properties could be useful for interaction with OTA. In a similar work which was done by Sargazi et al, dopamine loaded MNPs were synthesized to be utilized as a nanosized functional adsorbent in extraction of $\mathrm{OTA}^{22}$. Because in proper condition, the terminal amino group of dopamine has the positively charged $\left(-\mathrm{NH}_{3}^{+}\right)^{18,23}$ and can interact with OTA which exists in anionic form (OTA') ${ }^{24}$.

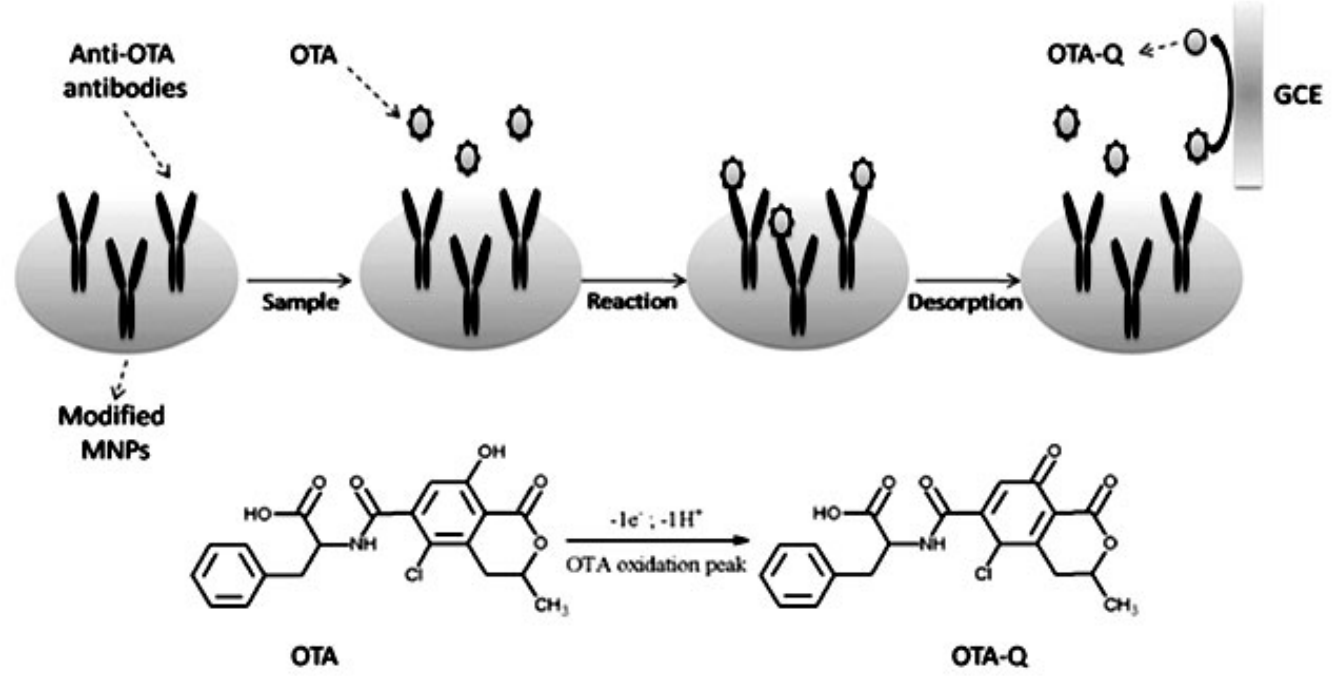

Fig. 3: Schematic representation of immunosensor preparation and also oxidation of OTA

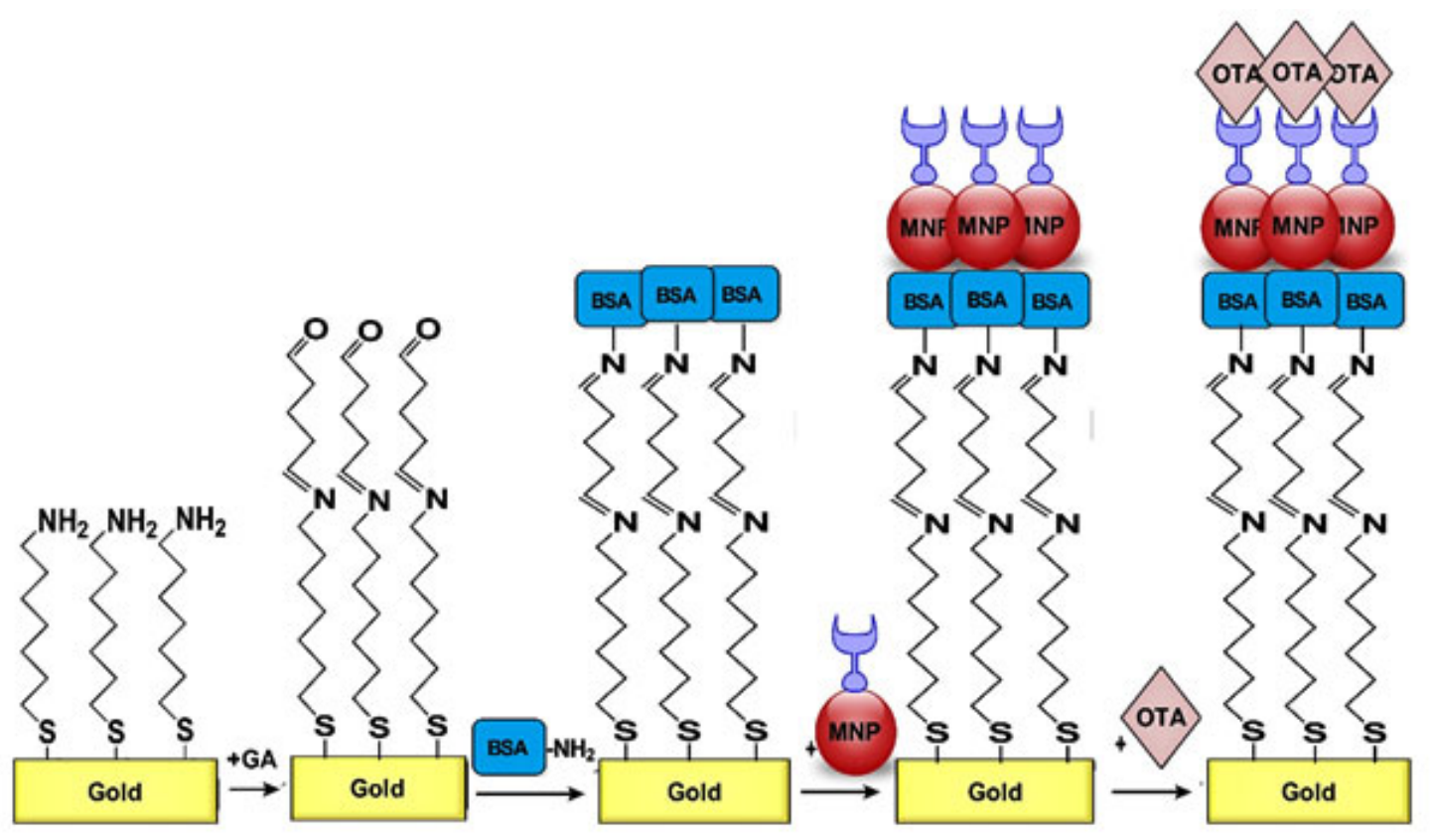

Fig. 4: The preparation steps of the label-free immunosensor based on MNPs 
After examination of different parameters which affect the efficiency of new adsorbents, the selectivity of magnetic sorbents for OTA in real samples such as cereal and rice were confirmed. Low detection limit (LOD $\left.<0.07 \mathrm{ng} \mathrm{mL}^{-1}\right)$, high precision $(\mathrm{RSD}<7 \%$ ) and good trueness (recoveries $>87 \%$ ) of tests showed that magnetic solid phase extraction, as an alternative cleanup method in comparison to immunoaffinity column procedure, possess the ability to extract OTA from real samples.

Magnetic Solid Phase Extraction by AptamerTargeted Magnetic Nanospheres

In this work the aptamers for OTA were immobilized onto the carboxyl group-modified magnetic nanospheres and were utilized for magnetic solid-phase extraction (MSPE) procedure. Figure 2 depicts the preparation of the magnetic nanospheres-aptamer (MNS-aptamer) adsorbents ${ }^{25}$.

Clean up by the MSPE can save the time of extraction, because MNPs can be readily collected by an external magnetic field, and also further purification such as centrifugation or filtration isn't necessary for this procedure ${ }^{26}$. Aptamers that bind to OTA, in the presence of $\mathrm{Mg}^{2+}$, show a G-quarter structure, and therefore, occur the special OTAaptamer interaction with high efficiency. Finally, the magnetic nanospheres (MNS) are collected using an external magnetic field, and quantified by HPLC.
The specificity of the magnetic aptamer toward OTA was examined in the presence of several compounds which have structural similarities to OTA. This test hinted that presence of other mycotoxins like zearalenone (ZEN) and deoxynivalenol (DON) have no impact on performance of MNS-aptamer toward ochratoxin A.

The MNS-aptamer sorbent, because of both short diffusion path and large surface area to volume ratio of MNPs ${ }^{27}$, shows high extraction capacity (500 ng OTA $/ \mathrm{mL}$ ); and it seems that development of method for detection of OTA in real food samples, be effective. Therefore, the ability of this method for real samples such as cereal and coffee, also was examined. The recovery amounts of OTA in three matrix samples were ranged from $67.2 \%$ to $90.4 \%$ and showed the acceptable affinity of sorbent to the target molecule.

All these results are confirmed that magnetic solid phase extraction by MNS-aptamer has the combined advantages of easy and inexpensive preparing in comparison to immunoaffinity columns.

Ochratoxin A Determination by an Electrochemical Method Using Modified Magnetic Nanoparticles

In this procedure for determination of OTA in food samples, an electrochemical technique was utilized where modified magnetic

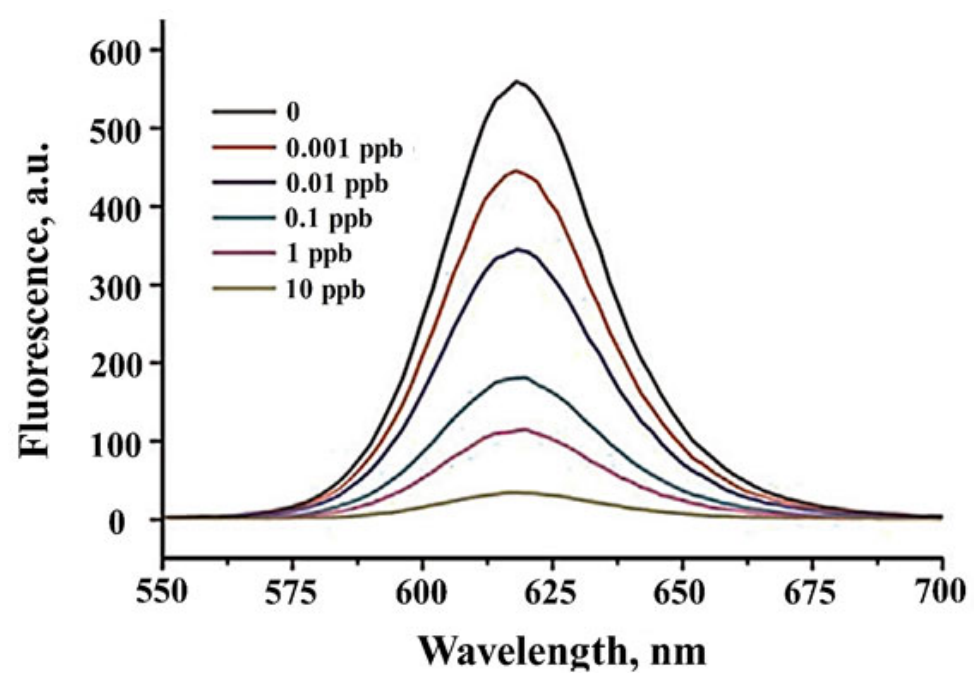

Fig. 5: The fluorescence spectrum of rapid OTA detection 
nanoparticles (MNPs) were combined with square wave voltammetry (SWV) ${ }^{28}$. Although, square wave voltammetry (SWV) hasn't proper detection limits for OTA determination, but physical properties of MNPs can enhance the analytical applications of SWV as a beneficial tool for detection of ochratoxin A ${ }^{28,29}$. Briefly, the surface of MNPs was modified by mouse monoclonal anti-OTA antibody to improve the performance of the SWV. For the pre-concentration and transfer into electrochemical cells, the OTA was adsorbed to the anti-OTA antibody and then was eluted by desorption solvent. Finally, existence of OTA was monitored by measuring the oxidation rate of it in acidic conditions via standard glassy carbon (GC) electrode (Fig. 3).
Due to the phenolic group of isocoumarin moiety and the carboxyl group of phenylalanine moiety in the OTA structure, it has weak acidic properties; so, an acidic solution composed of perchloric acid was used for desorption of OTA from MNPs. Because, according to $\mathrm{pH}$-partition theory, acids are un-ionized in acidic solutions and so the lowest amount of absorption occurs in the $\mathrm{pH}$ range from 1.0 to $5.0^{22}$. Since, this new method is inexpensive, reproducible and accurate, could be a good candidate for the determination of OTA in real samples in comparison with older methods.

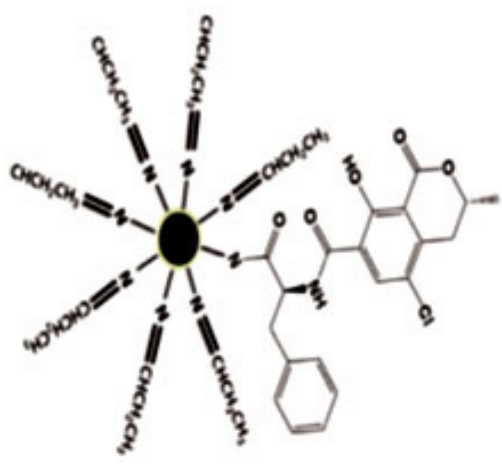

Step 1. Extraction of ochratoxin A on magnetic bead and capping amine groups

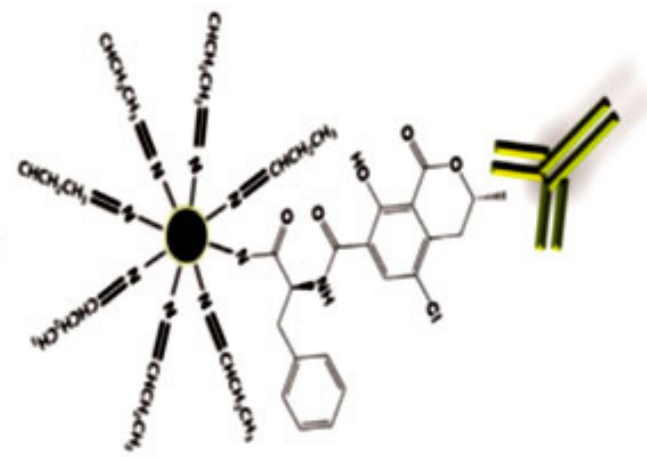

Step 2. Immunoreaction for tagging primary antibody

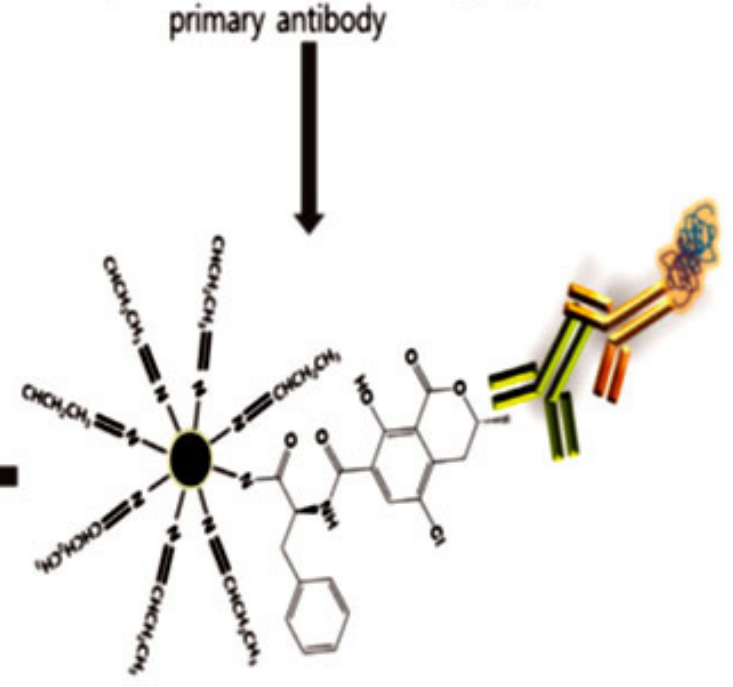

Step 3. HRP tagging for $\mathrm{CL}$ reaction

Step 4. Detection of OTA using the lab-built $\mathrm{CL}$ system

Fig. 6: Schematic steps for the detection of ochratoxin A using chemiluminescence system 
Use of Magnetic Nanoparticles in Detection of Ochratoxin A by a Label-Free Immunosensor

Nearly the same of before procedure, magnetic properties of MNPs are used for immobilization of anti-OTA mouse monoclonal antibody on external layer of gold electrode ${ }^{30}$. In order to reduce the direct binding of ochratoxin $\mathrm{A}$ to $\mathrm{Au}$ working electrode and prevention of direct oxidation at the naked electrode, the surface of electrode was covered utilizing bovine serum albumin (BSA) before attaching to MNPs ${ }^{31,32}$. Figure 4 represents all steps of the preparation of the immunosensor.

As a great advantage, The surface of biosensors can be renewed rapidly by an external magnet, when MNPs respond to the applied magnetic field ${ }^{33}$. Also, MNPs can provide several functional groups on the surface of electrode which lead to increasing the stability and number of the surface-bound antibodies ${ }^{34}$. On the other hand, electrochemical detection of OTA at $\mathrm{pH} \geq 7$ was simply possible, because molecule at $\mathrm{pH}$ above 7 shows both mono-anionic (OTA-) and di-anionic $\left(\mathrm{OTA}^{2-}\right)$ forms. Therefore, on the surface of electrode, all existing active sites related to the antibodies were occupied, when concentration was higher than $5 \mathrm{ng} /$ $\mathrm{mL}$ of OTA.

Cyclic voltammetry (CV) analysis represents that the bio-magnetic shell has promising reproducibility which is confirmed by standard deviation of $6.5 \%(n=5)$. According to quick and valid results plus sensitivity, accuracy and reproducibility of this magnetic immunosensor, authors have suggested it as an applicable bio-analytical tool for detection of OTA in real samples.

\section{Detection of Ochratoxin A by Combining Magnetic Nanoparticles and Rolling Circular Amplification}

ELISA (enzyme-linked immunosorbent assay) traditionally utilize the antibodies, but its storage is hard, and its preparation needs to long time and high $\cos ^{35}$. As a new technique with excellent sensitivity for detection of OTA, Yao et al combined the magnetic purification with the system of rolling circular amplification (RCA) to reduce the noise and to enhance the signal. Since the magnetic carriers have got functional groups like ion exchanger ligands, hydrophobic groups or bio-polymeric agents, the purification can be done in one step without utilizing centrifuges, filters or other equipment ${ }^{36}$. For this purpose, carboxyl-MNPs were prepared by solve-thermal protocol ${ }^{37}$ and were surface modified via the RCA primer containing the specific aptamer related to OTA ${ }^{38}$.

Sensitive detection of OTA was carried out using the above RCA system and also a QDs labeled ssDNA probe with fluorescent properties to improve sensitivity of system and to amplify signals. Figure 5 shows that by using of QDs, fluorescence intensity related to RCA system is decreased, when the concentration of OTA is increased. Of note, by purifying the residual QDs labeled probes through the magnetic separation operation, the interference of background fluorescent noise was reduced and thus efficiency and sensitivity for OTA detection were improved.

Theoretically, in this ultrasensitive detection, ochratoxin A combine with the primer and prevent from fluorescent emission, but in the absence of OTA, the primer onto magnetic nanoparticles would react with the RCA padlock which lead to strong fluorescent emission. So in general, this integrated platform could be useful for rapid detection of OTA in about $80 \mathrm{~min}$, and could be a viable alternative for traditional methods of OTA detection.

\section{Determination of Ochratoxin A by} Chemiluminescence Immunoassay Technique Using Magnetic Nanoparticles

In this technique, a chemiluminescence $(\mathrm{CL})$ immunoassay was coupled with amine-functionalized magnetic nanoparticles (MNPs) for chemically extraction and determination of OTA ${ }^{39}$. Generally for the extraction of OTA by MNPs, the carboxylic acid of OTA was activated by EDC (N-(3-dimethyl amino propyl)-N-ethyl carbodiimide hydrochloride) and NHS (N-hydroxysuccinimide) (Fig. 6, step 1) for directly reaction of the carboxylic group of OTA with amine-functionalized MNPs. Thereupon, for $\mathrm{CL}$ detection, monoclonal primary antibody and horseradish peroxidase (HRP) conjugated polyclonal secondary antibody were respectively reacted with the OTA-MNPs (Figure 6, step 2 and 3). When, this procedure was utilized for rice samples, the average limit of detection of $1.39 \mathrm{pg} / \mathrm{mL}\left(R^{2}=0.988\right)$ was obtained which confirmed this developed procedure 
as a useful tool for the examination of various toxins like OTA.

\section{CONCLUSION}

Detection of OTA in food samples is very critical because it can act as a potential carcinogen and neurotoxic. Up to now, scientists have done a lot of effort to assay OTA by different methods, but most of them have many disadvantages such as lack of selectivity and sensitivity or require to expensive apparatus and complex procedures. Recently, novel methods, as nanotechnologies, are combined with usual detection methods to advance them to higher levels. Our review confirmed that utilize of MNPs in some procedures such as aptamer-based immunosensor, chemiluminescence immunoassay, square wave voltammetry or even solid phase extraction can reduce the cost and facilitate the preparation. On the other hand, it could increase the stability of the antibodies on surface of sensors and also increase the speed, sensitivity, precision and reproducibility of these approaches.

\section{REFERENCES}

1. Binder, E. M. Animal feed science and technology. 2007, 133, 149-166

2. Duarte, S.; Pena, A.; Lino, C. Food Microbiology. 2010, 27, 187-198

3. Richard, J. L. International journal of food microbiology. 2007, 119, 3-10

4. Hussein, H. S.; Brasel, J. M. Toxicology. 2001, 167, 101-134

5. Bullerman, L. B.; Bianchini, A. International Journal of Food Microbiology. 2007, 119, 140-146

6. Ghali, R.; Hmaissia-Khlifa, K.; Ghorbel, H.; Maaroufi, K.; Hedili, A. Food Control. 2008, 19, 921-924

7. Gambacorta, S.; Solfrizzo, H.; Visconti, A.; Powers, S.; Cossalter, A.; Pinton, P.; Oswald, I. World Mycotoxin Journal. 2013, 6, 299-308

8. Li, S.; Marquardt, R.; Frohlich, A.; Vitti, T.; Crow, G. Toxicology and applied pharmacology. 1997, 145, 82-90

9. Heussner, A. H.; Bingle, L. E. Toxins. 2015, 7, 4253-4282

10. Ringot, D.; Chango, A.; Schneider, Y.-J.; Larondelle, Y. Chemico-biological interactions. 2006, 159, 18-46

11. Gilbert, J.; Brereton, P.; MacDonald, S. Food Additives \& Contaminants. 2001, 18, 10881093

12. Il'ichev, Y. V.; Perry, J. L.; Rüker, F.; Dockal, M.; Simon, J. D. Chemico-biological interactions. 2002, 141, 275-293

13. Wang, C.; Qian, J.; Wang, K.; Wang, K.; Liu, Q.; Dong, X.; Wang, C.; Huang, X. Biosensors and Bioelectronics. 2015, 68, 783-790

14. González-Peñas, E.; Leache, C.; Viscarret, M.;
De Obanos, A. P.; Araguás, C.; De Cerain, A. L. Journal of Chromatography A. 2004, 1025 , 163-168

15. Bazin, I.; Nabais, E.; Lopez-Ferber, M. Toxins. 2010, 2, 2230-2241

16. Wang, Q.; Chen, M.; Zhang, H.; Wen, W.; Zhang, X.; Wang, S. Analytical Methods. 2015, 7, 10224-10228

17. Xie, C.; Li, H.; Li, S.; Gao, S. Microchimica Acta. 2011, 174, 311-320

18. Sargazi, A.; Kuhestani, K.; Nosrat Nahoki, T.; Heidari Majd, M. International Journal of Pharmaceutical Sciences and Research. 2015, 6, 5047-5055

19. Heidari Majd, M.; Akbarzadeh, A.; Sargazi, A. Artificial Cells, Nanomedicine and Biotechnology. 2017, 45, 441-447.

20. Mashhadizadeh, M. H.; Amoli-Diva, M.; Pourghazi, K. Journal of Chromatography A. 2013, 1320, 17-26

21. Hackbart, H.; Prietto, L.; Primel, E. G.; GardaBuffon, J.; Badiale-Furlong, E. Journal of the Brazilian Chemical Society. 2012, 23, 103109

22. Sargazi, A.; Aliabadi, A.; Rahdari, A.; Allahdini-Hesaroiyeh, S.; Nejati-Yazdinejad, M.; Heidari Majd, M. Journal of the Brazilian Chemical Society. 2016, 1-10

23. Saei, A. A.; Barzegari, A.; Majd, M. H.; Asgari, D.; Omidi, Y. Journal of Nanoparticle Research. 2014, 16,

24. Poor, M.; Kunsagi-Mate, S.; Szente, L.; Matisz, G.; Secenji, G.; Czibulya, Z.; Koszegi, T. Food Chem. 2015, 172, 143-149

25. Wu, X.; Hu, J.; Zhu, B.; Lu, L.; Huang, X.; 
Pang, D. Journal of Chromatography A. 2011, 1218, 7341-7346

26. Han, Q.; Wang, Z.; Xia, J.; Chen, S.; Zhang, X.; Ding, M. Talanta. 2012, 101, 388-395

27. Jiao, Z.; Jiao, S.; Guo, Z.; Chen, H.; Zhang, N.; Huang, W. Food Analytical Methods. 2016, 1-7

28. Fernández-Baldo, M. A.; Bertolino, F. A.; Messina, G. A.; Sanz, M. I.; Raba, J. Talanta. 2010, 83, 651-657

29. Anjo, D. M.; Kahr, M.; Khodabakhsh, M.; Nowinski, S.; Wanger, M. Analytical chemistry. 1989, 61, 2603-2608

30. Zamfir, L.-G.; Geana, I.; Bourigua, S.; Rotariu, L.; Bala, C.; Errachid, A.; Jaffrezic-Renault, N. Sensors and Actuators B: Chemical. 2011, 159, 178-184

31. Perrotta, P. R.; Vettorazzi, N. R.; Arévalo, F. J.; Granero, A. M.; Chulze, S. N.; Zón, M. A.; Fernández, H. Electroanalysis. 2011, 23, 1585-1592
32. Ramírez, E. A.; Zón, M. A.; Ulloa, P. A. J.; Squella, J. A.; Vergara, L. N.; Fernández, H. Electrochimica Acta. 2010, 55, 771-778

33. Santandreu, M.; Solé, S.; Fàbregas, E.; Alegret, S. Biosensors and Bioelectronics. 1998, 13, 7-17

34. Yu, D.; Blankert, B.; Bodoki, E.; Bollo, S.; Viré, J.-C.; Sandulescu, R.; Nomura, A.; Kauffmann, J.-M. Sensors and Actuators B: Chemical. 2006, 113, 749-754

35. Zhao, Q.; Lv, Q.; Wang, H. Analytical chemistry. 2013, 86, 1238-1245

36. Safarik, I.; Safarikova, M. Biomagnetic Research and Technology. 2004, 2, 7

37. Liu, J.; Sun, Z.; Deng, Y.; Zou, Y.; Li, C.; Guo, X.; Xiong, L.; Gao, Y.; Li, F.; Zhao, D. Angewandte Chemie. 2009, 121, 5989-5993

38. Yao, L.; Chen, Y.; Teng, J.; Zheng, W.; Wu, J.; Adeloju, S. B.; Pan, D.; Chen, W. Biosensors and Bioelectronics. 2015, 74, 534-538

39. Kim, S.; Lim, H. Talanta. 2015, 140, 183-188 